\title{
]jfis
}

\section{Multi-objective Portfolio Selection Based on Skew-Normal Uncertainty Distribution and Asymmetric Entropy}

\author{
Seyyed Hamed Abtahi ${ }^{1}$, Gholamhossein Yari $^{2}$, Farhad Hosseinzadeh Lotfi ${ }^{1}$, \\ and Rahman Farnoosh ${ }^{2}$ \\ ${ }^{1}$ Department of Statistics, Science and Research Branch, Islamic Azad University, Tehran, Iran \\ ${ }^{2}$ Department of Mathematics, Iran University of Science and Technology, Tehran, Iran
}

\begin{abstract}
Empirical studies illustrate that in numerous cases the returns of securities are not normally distributed. In this paper, skew-normal uncertainty distribution is proposed to capture skewness in the portfolio selection problem. Furthermore, the concept of asymmetric entropy for uncertain variables as the quantifier of diversification is presented and its mathematical properties such as translation invariance and positive linearity are studied. To examine the effect of asymmetric entropy parameter on portfolio diversification, a mean-CVaR-entropy portfolio selection problem is presented based on asymmetric entropy with different parameter values and logarithm entropy. A non-dominated sorting genetic algorithm II (NSGA-II) is implemented in MATLAB to solve the corresponding problem. Numerical results show that asymmetric entropy for a specific parameter value will outperform logarithm entropy in portfolio diversification.
\end{abstract}

Keywords: Uncertain variable, Skew-normal uncertainty distribution, Asymmetric entropy, Mean-CVaR-entropy portfolio selection model, Non-dominated sorting genetic algorithms II (NSGA-II)

\section{Introduction}

The financial crisis of 2007-2008 showed that rare and unpredictable events can challenge conventional ideas about portfolio construction. However, we do not know in advance when such events will occur and how traumatic they can be, we can modify current risk management frameworks to better manage these rare and dangerous events.

The prime reason for the occurrence of such anomalies lies in the conventional approach to applying mean-variance model which was introduced by Markowitz [1] in 1952. The basic assumption in the mean-variance model and many other models is that future returns will be independent and normally distributed. Sheikh and Qiao [2] declared that in many cases it can be empirically observed that returns are not normally distributed and under non- normality, variance becomes inefficient as the quantifier of portfolio risk.

Most of studies on portfolio selection have been down based on only the first two moments of return distributions. However, there has always been a contentious issue whether higher moments should be considered in portfolio selection. Arditti [3] and Arditti and Levy [4] declared that higher moments should be considered for non-normal returns. 
Skewness as the third central moment was defined to measure asymmetry of return distributions. Chunhachinda et al. [5] studied international stock market and confirmed that higher moments cannot be neglected in the portfolio selection. Konno et al. [6] constructed a portfolio optimization model by applying skewness. Keel et al. [7] showed that presence of skewness has a significant influence on portfolio selection. Furthermore, to better capture skewness, some scholars applied different distributions in portfolio optimization. Hu and Kercheval [8] argued that $t$ and skewed $t$ distribution show a much better fit to real data than the normal distribution.

Several portfolio selection models are developed by considering more efficient risk measure such as value at risk (VaR), conditional value at risk (CVaR) and entropy. VaR better reflects extreme events, but it does not aggregate risk in the sense of being sub-additive on portfolios [9]. CVaR is a coherent risk measure which is a superior to VaR [10]. VaR and CVaR has been widely applied by researchers [11-16].

Entropy of random variables first proposed by Shannon [17] in logarithm form. The study carried out by Philippatos and Wilson [18] was the pioneering work to associate entropy with a measure of risk in portfolio selection. They argued that entropy is more general and better suited for the selection of portfolios than variance. Furthermore, Simonelli [19] showed that entropy as a measure of risk is better than variance in wealth allocation.

According to the knowledge of securities investment, when the investment portfolio is more diversified, the risk of investment will decline. Entropy as a measure of diversification, originally developed by Jacquemin and Berry [20]. Entropy is a widely accepted measure of diversity [21] and has major advantages in the analysis of portfolio diversification [20]. It is well-established that the greater the level of entropy, the higher the degree of portfolio diversification. Bera and Park [22] constructed a well-diversified portfolio based on maximum entropy principle.

In the mentioned literatures it is assumed that the security returns are random variable with probability distribution. The fundamental assumption for using probability theory in the portfolio selection is that the probability distribution of security returns is similar to the past one and close enough to frequencies. However, it is difficult to ensure this assumption. In financial businesses, sometimes we have historical data scarcity. Therefore, we ask domain experts to evaluate the belief degree that each event will happen. Using fuzzy set theory is a way to handle portfolio optimization problems with returns given by experts' evaluations. Liu [23] presented a counterexam- ple to show that modeling belief degree which uses subjective probability may lead to counterintuitive results. Furthermore, Liu [24] showed that fuzzy set theory is not self-consistent in mathematics and may lead to wrong results in practice. The main mistake of fuzzy set theory is based on the wrong assumption that the belief degree of a union of events is the maximum of the belief degrees of the individual events no matter if they are independent or not.

In order to better deal with belief degree, uncertainty theory was founded by Liu [25] in 2007. Uncertainty theory has been employed in the portfolio optimization researches. Zhai et al. [26] formulated an uncertain multi-objective portfolio optimization considering skewness. Bhattacharyya et al. [27] studied effects of skewness on the portfolio selection. Yan [28] presented mean-VaR optimization model under uncertainty. Ning et al. [29] proposed mean-TVaR model under uncertainty framework.

Liu [30] proposed a concept of entropy for uncertain variables in the form of logarithm function. Chen and Dia [31] proposed the maximum entropy principle for uncertain variables. Then, Dai and Chen [32] presented a formula for calculating the entropy of function of uncertain variables. Entropy as a quantitative estimate of diversity, has been applied in portfolio selection [33].

There are a large number of optimization problems that have more than one objective. In some cases, the optimization problem is formulated to maximize one objective while minimize the other one. Numerous multi-objective optimization problems have been proposed. Deb et al. [34] proposed non-dominated sorting genetic algorithm II (NSGA-II) for multi-objective optimization problem. Kennedy and Eberhart [35] proposed particle swarm optimization (PSO) algorithm which is used to simulate the social behaviors of animals. PSO lies somewhere between genetic and evolutionary algorithms. Fonseca and Fleming [36] introduced multi-objective genetic algorithms (MOGAs). Ziztler et al. [37] carried out comparison of several evolutionary algorithms and discussed some results. Sherinov and Unveren [38] proposed multi-objective imperialistic competitive algorithm (MOICA) which is multi-objective version of imperialist competitive algorithm (IAC) introduced by Atashpaz-Gargari and Lucas [39]. The authors concluded that ICA and GA (genetic algorism) algorithms reach the global solution in the same iterations unlike PSO algorithm which needs more.

In this research, after providing definition of key terms such as skew-normal uncertainty distribution and asymmetric entropy 
and stating their properties in Sections 2 and 3, mean-CVaRentropy model based on skew-normal uncertain variables and asymmetric entropy with different parameter values will be discussed in Section 4. Then in Section 5, a numerical example is put forth and finally in Section 6, conclusions and suggestions are presented.

\section{Preliminaries}

Uncertainty theory is a branch of mathematics and was founded by Liu [25] in 2007. Having sample scarcity, we should ask experts to evaluate the degree of belief in the occurrence of an event. Modeling belief degree which uses subjective probability or fuzzy set theory may lead to counterintuitive results. Therefore, we use uncertainty theory to model belief degree. This section comes with reviewing some necessary definitions and theorems.

Assume that $\Gamma$ is a nonempty set and $\mathcal{L}$ represents a $\sigma$-algebra over $\Gamma$. Each element $\Lambda$ in $\mathcal{L}$ is an event. Liu [25] presented an axiomatic uncertain measure $\mathcal{M}(\Lambda)$ to indicate the belief degree that uncertain event $\Lambda$ occurs, where the uncertain measure $\mathcal{M}: \mathcal{L} \rightarrow[0,1]$ satisfies the following three axioms:

Axiom 1 (Normality). $\mathcal{M}(\Gamma)=1$ for the universal set $\Gamma$.

Axiom 2 (Duality). $\mathcal{M}\{\Lambda\}+\mathcal{M}\left\{\Lambda^{c}\right\}=1$ for any event $\Lambda$, where $\Lambda^{c}$ is the complementary set of $\Lambda$.

Axiom 3 (Subadditivity). For every countable sequence of events $\Lambda_{1}, \Lambda_{2}, \ldots$, we have

$$
\mathcal{M}\left\{\bigcup_{i=1}^{\infty} \Lambda_{i}\right\} \leq \sum_{i=1}^{\infty} \mathcal{M}\left\{\Lambda_{i}\right\}
$$

the triplet $(\Gamma, \mathcal{L}, \mathcal{M})$ is called an uncertainty space.

The product uncertain measure $\mathcal{M}$ on the product $\sigma$-algebra $\Gamma$ is defined by Liu [30] as the following product axiom:

Axiom 4 (Product). Let $\left(\Gamma_{k}, \mathcal{L}_{k}, \mathcal{M}_{k}\right)$ be uncertainty spaces for $K=1,2, \ldots$. The product uncertain measure $\mathcal{M}$ is an uncertain measure satisfying

$$
\mathcal{M}\left\{\prod_{k=1}^{\infty} \Lambda_{k}\right\}=\bigwedge_{k=1}^{\infty} \mathcal{M}_{k}\left\{\Lambda_{k}\right\}
$$

where $\Lambda_{k}$ are arbitrary chosen events from $\mathcal{L}_{k}$ for $k=1,2, \ldots$, respectively.

Definition 1 (Liu [25]). The uncertainty distribution of an uncertain variable $\xi$ is defined as follows:

$$
\Phi(x)=\mathcal{M}\{\xi \leq x\}
$$

for any real number $x$.

Theorem 1 (Liu and Lio [40]). A real-valued function $\Phi(x)$ on $\mathfrak{R}$ is an uncertainty distribution if and only if it is a monotone increasing function satisfying

$$
\begin{aligned}
& 0 \leq \Phi(x) \leq 1, \Phi(x) \not \equiv 0, \Phi(x) \not \equiv 1, \\
& \Phi\left(x_{0}\right)=1 \text { if } \Phi(x)=1 \text { for any } x>x_{0} .
\end{aligned}
$$

Definition 2 (Liu [41]). An uncertain variable $\xi$ is called normal denoted by $N(e, \sigma)$ if it has a normal uncertainty distribution

$$
\Phi(x)=\left(1+\exp \left(\frac{\pi(e-x)}{\sqrt{3} \sigma}\right)\right)^{-1} ; x \in \mathbb{R},
$$

where $e$ and $\sigma(\sigma>0)$ are real numbers.

Definition 3. An uncertain variable $\xi$ is called skew-normal denoted by $S N(e, p, \sigma)$ if it has a skew-normal uncertainty distribution

$$
\Phi(x)=\left(1+\exp \left(\frac{\pi(e-x)}{\sqrt{3} \sigma}\right)\right)^{-p} ; x \in \mathbb{R},
$$

where $e, \sigma(\sigma>0)$ and $p(p>0)$ are real numbers.

Remark 1. Suppose that $\xi \sim S N(e, p, \sigma)$. Parameter $p$ specifies distribution shape as follows:

1. If $p=1$, then an uncertain variable $\xi$ is normal distribution.

2. If $p>1$, then an uncertain variable $\xi$ is positive skewnormal distribution.

3. If $0<p<1$, then an uncertain variable $\xi$ is negative skew-normal distribution.

In order to estimate the parameter vector $\Theta=(e, p, \sigma)$, we employ the least squares principle proposed by Liu [42] to minimize the sum of squares of the distance between the expert's experimental data and the uncertainty distribution. assume that the uncertainty distribution to be determined has a function form $\Phi(x \mid \Theta)$ with an unknown parameter vector $\Theta=(e, p, \sigma)$. The optimal solution vector $\widehat{\Theta}=(\hat{e}, \hat{p}, \widehat{\sigma})$ of $\operatorname{Min} \sum_{i=1}^{n}\left(\Phi(x \mid \Theta)-\alpha_{i}\right)^{2}$ is called the least squares estimate of the parameter vector $\Theta=(e, p, \sigma)$.

Theorem 2. Let $\Phi(x \mid \Theta)$ be a skew-normal uncertainty distribution with parameter vector $\Theta=(e, p, \sigma)$. If the expert's experimental data $(\boldsymbol{x}, \boldsymbol{\alpha})=\left(x_{1}, \alpha_{1}\right),\left(x_{2}, \alpha_{2}\right), \ldots,\left(x_{n}, \alpha_{n}\right)$ are determined, then the least squares estimate of parameter 
vector $\Theta=(e, p, \sigma)$ is obtained by solving following equations:

$$
\left\{\begin{array}{rl}
-2 & p \sum_{i=1}^{n}\left[\frac{\pi}{\sqrt{3} \sigma} \exp \left(\frac{\pi\left(e-x_{i}\right)}{\sqrt{3} \sigma}\right)\right. \\
& \times\left(1+\exp \left(\frac{\pi\left(e-x_{i}\right)}{\sqrt{3} \sigma}\right)\right)^{-p-1} \\
& \left.\times\left(\left(1+\exp \left(\frac{\pi\left(e-x_{i}\right)}{\sqrt{3} \sigma}\right)\right)^{-p}-\alpha_{i}\right)\right]=0 \\
\frac{2 p}{\sigma} & \sum_{i=1}^{n}\left[\frac{\pi}{\sqrt{3} \sigma}\left(e-x_{i}\right) \exp \left(\frac{\pi\left(e-x_{i}\right)}{\sqrt{3} \sigma}\right)\right. \\
& \left.\times\left(1+\exp \left(\frac{\pi\left(e-x_{i}\right)}{\sqrt{3} \sigma}\right)\right)^{-p-1}\right) \\
& \left.\times\left(\left(1+\exp \left(\frac{\pi\left(e-x_{i}\right)}{\sqrt{3} \sigma}\right)\right)^{-p}-\alpha_{i}\right)\right]=0 \\
-2 & \sum_{i=1}^{n}\left[\left(1+\exp \left(\frac{\pi\left(e-x_{i}\right)}{\sqrt{3} \sigma}\right)\right)^{-p}\right. \\
& \left.\left.\times \ln \left(1+\exp \left(\frac{\pi\left(e-x_{i}\right)}{\sqrt{3} \sigma}\right)\right)^{-p}-\alpha_{i}\right)\right]=0 \\
& \times\left(1+\exp \left(\frac{\pi\left(e-x_{i}\right)}{\sqrt{3} \sigma}\right)\right)^{-p}
\end{array}\right.
$$

Proof. Let $L=\sum_{i=1}^{n}\left[\left(\left(1+\exp \left(\frac{\pi\left(e-x_{i}\right)}{\sqrt{3} \sigma}\right)\right)^{-p}-\alpha_{i}\right)^{2}\right]$. By calculating derivatives of $\mathrm{L}$ with respect to $e, \sigma$ and $p$ (i.e., $\frac{\partial L}{\partial e}, \frac{\partial L}{\partial \sigma}$ and $\left.\frac{\partial L}{\partial p}\right)$ and setting them equal to zero, equations are obtained.

Theorem 3 (Liu 2013). A function $\Phi^{-1}(r):(0,1) \rightarrow \mathbb{R}$ is an inverse uncertainty distribution if and only if it is a continuous and strictly increasing function with respect to $r$.

Theorem 4. Let $\xi \sim S N(e, p, \sigma)$, then the inverse uncertainty distribution of skew-normal uncertain variable $\xi$ is

$$
\Phi^{-1}(r)=e+\frac{\sqrt{3} \sigma}{\pi} \ln \left(\frac{r^{\frac{1}{p}}}{1-r^{\frac{1}{p}}}\right) ; 0<r<1
$$

Theorem 5 (Liu [41]). Let $\xi_{1}, \xi_{2}, \ldots, \xi_{n}$ be independent uncertain variables with regular uncertainty distributions $\Phi_{1}$, $\Phi_{2}, \ldots, \Phi_{n}$, respectively. If $f\left(\xi_{1}, \xi_{2}, \ldots, \xi_{n}\right)$ is strictly increasing with respect to $\xi_{1}, \xi_{2}, \ldots, \xi_{m}$ and strictly decreasing with respect to $\xi_{m+1}, \xi_{m+2}, \ldots, \xi_{n}$, then

$$
\xi=f\left(\xi_{1}, \xi_{2}, \ldots, \xi_{n}\right)
$$

has an inverse uncertainty distribution

$$
\begin{aligned}
\Psi^{-1}(r)= & \left(\Phi_{1}^{-1}(r), \ldots, \Phi_{m}^{-1}(r), \Phi_{m+1}^{-1}(1-r), \ldots\right. \\
& \left.\Phi_{n}^{-1}(1-r)\right)
\end{aligned}
$$

Theorem 6. Let $\xi_{i} \sim S N\left(e_{i}, p_{i}, \sigma_{i}\right) ; \forall i=1,2, \ldots, n$, then the expected value of skew-normal uncertain variable $\xi_{i}$ is

$$
E\left[\xi_{i}\right]=e_{i}-\frac{\sqrt{3} \sigma_{i}}{\pi p_{i}}-\frac{\sqrt{3} \sigma_{i}}{\pi} \int_{0}^{1} \ln \left(1-r^{\frac{1}{p_{i}}}\right) d r .
$$

Definition 4 (Peng [10]). Let $\xi$ be an uncertain variable and $\alpha \in(0,1]$ be the risk confidence level. Then, the value at risk of $\xi$ at the confidence level $\alpha$, written as $\operatorname{VaR}_{\xi}(\alpha)$, is the function $\operatorname{VaR}_{\xi}(\alpha):(0,1] \rightarrow \mathbb{R}$ such that

$$
\operatorname{VaR}_{\xi}(\alpha)=\inf \{x \mid \mathcal{M}\{\xi \leq x\} \geq \alpha\}
$$

Definition 5 (Peng [10]). Let $\xi$ be an uncertain variable and $\alpha \in(0,1]$ be the risk confidence level. Then, the conditional value at risk of $\xi$ at the confidence level $\alpha$, written as $\mathrm{CVaR}_{\xi}(\alpha)$, is the function $\mathrm{CVaR}_{\xi}(\alpha):(0,1] \rightarrow \mathbb{R}$ such that

$$
\operatorname{CVaR}_{\xi}(\alpha)=\frac{1}{1-\alpha} \int_{\alpha}^{1} \operatorname{VaR}_{\xi}(\beta) d \beta
$$

\section{Asymmetric Entropy}

The purpose of this section is to present a new type of entropy called asymmetric entropy for uncertain variables for regular distributions. We first recall the concept of logarithm entropy proposed by Liu [30].

Definition 6 (Liu [30]). Suppose that $\xi$ is an uncertainty variable with uncertainty distribution $\Phi$. Then the logarithm entropy is defined by

$$
H[\xi]=\int_{-\infty}^{+\infty} \mathrm{L}(\Phi(x)) d x
$$

where $\mathrm{L}(\mathrm{t})=-\mathrm{t} \ln \mathrm{t}-(1-\mathrm{t}) \ln (1-\mathrm{t})$.

Definition 7. Suppose that $\xi$ is an uncertainty variable with uncertainty distribution $\Phi$. Then asymmetric entropy is defined by

$$
H[\xi]=\int_{-\infty}^{+\infty} \mathrm{S}(\Phi(x)) d x
$$

where $S(t)=\left(\frac{1}{3}\right)\left(-q \sqrt{t} \ln (t)-\left(\frac{1}{q}\right) \sqrt{1-t} \ln (1-t)\right) ; q>$ 0 . 


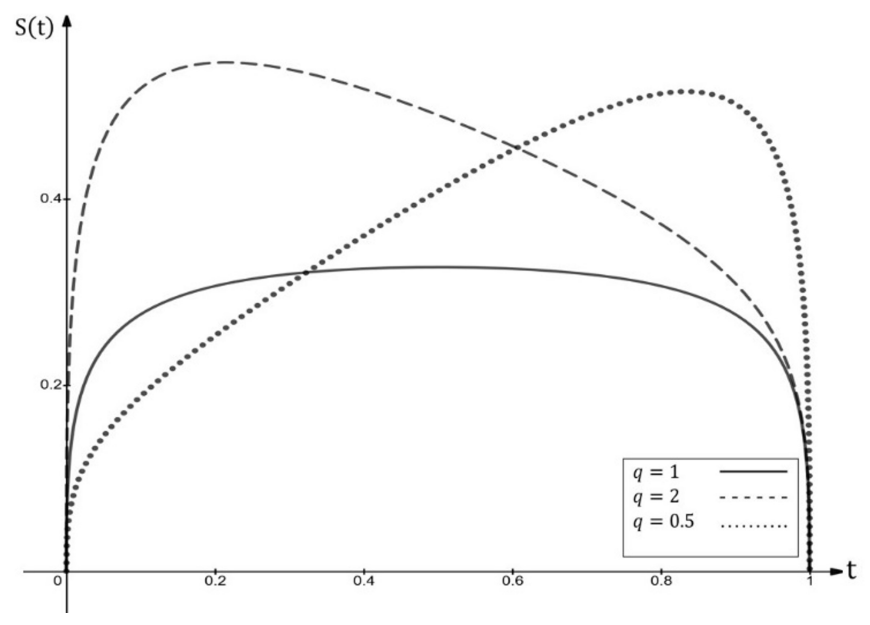

Figure 1. The function $\mathrm{S}(\mathrm{t})$ for different parameter $\mathrm{q}$.

Remark 2. For function $\mathrm{S}(t)$ (Figure 1):

1. If $q=1$, then $\mathrm{S}(\mathrm{t})$ is symmetric or zero skew.

2. If $q>1$, then $\mathrm{S}(\mathrm{t})$ is positive skew.

3. If $0<q<1$, then $\mathrm{S}(\mathrm{t})$ is negative skew.

Theorem 7. Suppose that $\xi$ is an uncertainty variable with regular uncertainty distribution $\Phi$. Then

$$
\begin{aligned}
H[\xi]= & -\int_{0}^{1} \Phi^{-1}(r)\left(\frac{1}{3}\right)\left(-\frac{\mathrm{q} \ln (\mathrm{r})}{2 \sqrt{\mathrm{r}}}-\frac{\mathrm{q}}{\sqrt{\mathrm{r}}}+\frac{\ln (1-\mathrm{r})}{2 \mathrm{q} \sqrt{1-\mathrm{r}}}\right. \\
& \left.+\frac{1}{\mathrm{q} \sqrt{1-\mathrm{r}}}\right) \mathrm{dr},
\end{aligned}
$$

where $q>0$.

Proof. It is clear that

$$
\begin{aligned}
S(\mathrm{r})= & \left(\frac{1}{3}\right)\left(-\mathrm{q} \sqrt{\mathrm{r}} \ln (\mathrm{r})-\left(\frac{1}{\mathrm{q}}\right) \sqrt{1-\mathrm{r}} \ln (1-\mathrm{r})\right) ; \\
& r m q>0
\end{aligned}
$$

is a derivable function whose derivative has the form

$$
S^{\prime}(\mathrm{r})=\left(\frac{1}{3}\right)\left(-\frac{\mathrm{q} \ln (\mathrm{r})}{2 \sqrt{\mathrm{r}}}-\frac{\mathrm{q}}{\sqrt{\mathrm{r}}}+\frac{\ln (1-\mathrm{r})}{2 \mathrm{q} \sqrt{1-\mathrm{r}}}+\frac{1}{\mathrm{q} \sqrt{1-\mathrm{r}}}\right)
$$$$
\mathrm{q}>0 \text {. }
$$

Since,

$$
S(\Phi(x))=\int_{0}^{\Phi(x)} S^{\prime}(\mathrm{r}) \mathrm{dr}=-\int_{\Phi(x)}^{1} S^{\prime}(\mathrm{r}) \mathrm{d} r
$$

we have:

$$
H[\xi]=\int_{-\infty}^{+\infty} S(\Phi(x)) \mathrm{d} x
$$

$$
=\int_{-\infty}^{0} \int_{0}^{\Phi(x)} S^{\prime}(\mathrm{r}) \operatorname{drd} x-\int_{0}^{+\infty} \int_{\Phi(x)}^{1} S^{\prime}(\mathrm{r}) \operatorname{drd} x .
$$

It follows from Fubini theorem that

$$
\begin{aligned}
H[\xi]= & \int_{0}^{\Phi(0)} \int_{\Phi^{-1}(\mathrm{r})}^{0} S^{\prime}(\mathrm{r}) \mathrm{d} x \mathrm{dr}-\int_{\Phi(0)}^{1} \int_{0}^{\Phi^{-1}(\mathrm{r})} S^{\prime}(\mathrm{r}) \mathrm{d} x \mathrm{dr} \\
= & -\int_{0}^{\Phi(0)} \Phi^{-1}(\mathrm{r}) S^{\prime}(\mathrm{r}) \mathrm{dr}-\int_{\Phi(0)}^{1} \Phi^{-1}(\mathrm{r}) S^{\prime}(\mathrm{r}) \mathrm{dr} \\
= & -\int_{0}^{1} \Phi^{-1}(\mathrm{r}) S^{\prime}(\mathrm{r}) \mathrm{dr} \\
= & -\int_{0}^{1} \Phi^{-1}(\mathrm{r})\left(\frac{1}{3}\right)\left(-\frac{\mathrm{q} \ln (\mathrm{r})}{2 \sqrt{\mathrm{r}}}-\frac{\mathrm{q}}{\sqrt{\mathrm{r}}}+\frac{\ln (1-\mathrm{r})}{2 \mathrm{q} \sqrt{1-\mathrm{r}}}\right. \\
& \left.+\frac{1}{q \sqrt{1-r}}\right) \mathrm{dr} .
\end{aligned}
$$

The theorem is verified.

Theorem 8. Let $\xi_{1}, \xi_{2}, \ldots, \xi_{n}$ be independent uncertain variables with regular uncertainty distributions $\Phi_{1}, \Phi_{2}, \ldots, \Phi_{n}$, respectively. If $f\left(\xi_{1}, \xi_{2}, \ldots, \xi_{n}\right)$ is strictly increasing with respect to $\xi_{1}, \xi_{2}, \ldots, \xi_{m}$ and strictly decreasing with respect to $\xi_{m+1}, \xi_{m+2}, \ldots, \xi_{n}$, then

$$
\xi=f\left(\xi_{1}, \xi_{2}, \ldots, \xi_{n}\right)
$$

has an entropy

$$
\begin{aligned}
H[\xi]= & -\int_{0}^{1} f\left(\Phi_{1}^{-1}(\mathrm{r}), \ldots, \Phi_{m}^{-1}(\mathrm{r}), \Phi_{m+1}^{-1}(1-\mathrm{r}), \ldots,\right. \\
& \left.\Phi_{n}^{-1}(1-\mathrm{r})\right)\left(\frac{1}{3}\right)\left(-\frac{\mathrm{q} \ln (\mathrm{r})}{2 \sqrt{\mathrm{r}}}-\frac{\mathrm{q}}{\sqrt{\mathrm{r}}}+\frac{\ln (1-\mathrm{r})}{2 \mathrm{q} \sqrt{1-\mathrm{r}}}\right. \\
& \left.+\frac{1}{q \sqrt{1-r}}\right) \mathrm{dr} .
\end{aligned}
$$

Proof. Since $f\left(x_{1}, x_{2}, \ldots, x_{n}\right)$ is strictly increasing with respect to $x_{1}, x_{2}, \ldots, x_{m}$ and strictly decreasing with respect to $x_{m+1}, x_{m+2}, \ldots, x_{n}$, it follows from Theorem 5 that the inverse uncertainty distribution of $\xi$ is

$$
\begin{aligned}
\Psi^{-1}(r)= & f\left(\Phi_{1}^{-1}(\mathrm{r}), \ldots, \Phi_{m}^{-1}(\mathrm{r}), \Phi_{m+1}^{-1}(1-\mathrm{r}), \ldots\right. \\
& \left.\Phi_{n}^{-1}(1-\mathrm{r})\right)
\end{aligned}
$$

By applying Theorem 7, the entropy formula is obtained.

Theorem 9 (Translation invariance). Let $\xi$ be an uncertain variable, and $c$ be a real number. Then,

$$
H[\xi+c]=H[\xi]
$$

Proof. Suppose that $\xi$ has an uncertainty distribution $\Phi$, i.e., 
$\Phi(x)=\mathcal{M}\{\xi \leq x\}$. Then, the uncertain variable $\xi+c$ has an uncertain distribution $(x)=\mathcal{M}\{\xi+c \leq x\}=\mathcal{M}\{\xi \leq$ $x-c\}=\Phi(x-c)$. By the definition of asymmetric entropy we have:

$$
\begin{aligned}
H[\xi+c] & =\int_{-\infty}^{+\infty} S(\Psi(x)) d x=\int_{-\infty}^{+\infty} S(\Phi(x-c)) d x \\
& =\int_{-\infty}^{+\infty} S(\Phi(x)) d x=H[\xi] .
\end{aligned}
$$

Theorem 10 (Positive linearity). Let $\xi$ and $\eta$ be independent uncertain variables. Then for any numbers $a \geq 0, b \geq 0$ and $q>0$ we have:

$$
H[a \xi+b \eta]=a H[\xi]+b H[\eta] .
$$

And for any numbers $a<0, b<0$ and $q=1$, we have:

$$
H[a \xi+b \eta]=|a| H[\xi]+|b| H[\eta] .
$$

Proof. Suppose that $\xi$ and $\eta$ have uncertainty distributions $\Phi$ and respectively. The theorem will be proved via three steps.

Step 1. We prove $H[a \xi]=a H[\xi]$.

If $a>0$, then the uncertain variable $a \xi$ has an inverse uncertain distribution $\Upsilon^{-1}(r)=a \Phi^{-1}(r)$.

By Theorem 7 we have:

$$
\begin{aligned}
H[a \xi]= & -\int_{0}^{1} a \Phi^{-1}(r)\left(\frac{1}{3}\right)\left(-\frac{\mathrm{q} \ln (\mathrm{r})}{2 \sqrt{\mathrm{r}}}-\frac{\mathrm{q}}{\sqrt{\mathrm{r}}}+\frac{\ln (1-\mathrm{r})}{2 \mathrm{q} \sqrt{1-\mathrm{r}}}\right. \\
& \left.+\frac{1}{\mathrm{q} \sqrt{1-\mathrm{r}}}\right) \mathrm{dr} \\
= & -a \int_{0}^{1} \Phi^{-1}(r)\left(\frac{1}{3}\right)\left(-\frac{\mathrm{q} \ln (\mathrm{r})}{2 \sqrt{\mathrm{r}}}-\frac{\mathrm{q}}{\sqrt{\mathrm{r}}}+\frac{\ln (1-\mathrm{r})}{2 \mathrm{q} \sqrt{1-\mathrm{r}}}\right. \\
& \left.+\frac{1}{\mathrm{q} \sqrt{1-\mathrm{r}}}\right) \mathrm{dr}=a H[\xi] .
\end{aligned}
$$

If $a=0$, then $H[a \xi]=0=a H[\xi]$.

If $a<0$, then the uncertain variable $a \xi$ has an inverse uncertain distribution $\Upsilon^{-1}(r)=a \Phi^{-1}(1-r)$.

By Theorem 7 we have:

$$
\begin{aligned}
H[a \xi]= & -\int_{0}^{1} a \Phi^{-1}(1-r)\left(\frac{1}{3}\right)\left(-\frac{\mathrm{q} \ln (\mathrm{r})}{2 \sqrt{\mathrm{r}}}-\frac{\mathrm{q}}{\sqrt{\mathrm{r}}}\right. \\
& \left.+\frac{\ln (1-\mathrm{r})}{2 \mathrm{q} \sqrt{1-\mathrm{r}}}+\frac{1}{\mathrm{q} \sqrt{1-\mathrm{r}}}\right) \mathrm{dr}
\end{aligned}
$$

by changing the variable $u=1-r$ we have:

$$
H[a \xi]=a \int_{0}^{1} \Phi^{-1}(u)\left(\frac{1}{3}\right)\left(-\frac{\mathrm{q} \ln (1-\mathrm{u})}{2 \sqrt{1-\mathrm{u}}}-\frac{\mathrm{q}}{\sqrt{1-\mathrm{u}}}\right.
$$

$$
\left.+\frac{\ln (\mathrm{u})}{2 \mathrm{q} \sqrt{\mathrm{u}}}+\frac{1}{\mathrm{q} \sqrt{\mathrm{u}}}\right) d u
$$

if $q=1$, then it can be proved that $S^{\prime}(1-u)=-S^{\prime}(u)$, thus:

$$
\begin{aligned}
H[a \xi]= & -a \int_{0}^{1} \Phi^{-1}(u)\left(\frac{1}{3}\right)\left(-\frac{\mathrm{q} \ln (\mathrm{u})}{2 \sqrt{\mathrm{u}}}-\frac{\mathrm{q}}{\sqrt{\mathrm{u}}}\right. \\
& \left.+\frac{\ln (1-\mathrm{u})}{2 \mathrm{q} \sqrt{1-\mathrm{u}}}+\frac{1}{\mathrm{q} \sqrt{1-\mathrm{u}}}\right) \mathrm{du} \\
= & |a| H[\xi] .
\end{aligned}
$$

Therefore, we have $H[a \xi]=|a| H[\xi]$.

Step 2. We prove that $H[\xi+\eta]=H[\xi]+H[\eta]$. The Inverse uncertainty distribution of $\xi+\eta$ is

$$
\Upsilon^{-1}(r)=\Phi^{-1}(r)+\Psi^{-1}(r),
$$

it follows from Theorem 7 that

$$
\begin{aligned}
H[\xi+\eta]= & -\int_{0}^{1}\left(\Phi^{-1}(r)+\Psi^{-1}(r)\right)\left(\frac{1}{3}\right)\left(-\frac{\mathrm{q} \ln (\mathrm{r})}{2 \sqrt{\mathrm{r}}}\right. \\
& \left.-\frac{\mathrm{q}}{\sqrt{\mathrm{r}}}+\frac{\ln (1-\mathrm{r})}{2 \mathrm{q} \sqrt{1-\mathrm{r}}}+\frac{1}{\mathrm{q} \sqrt{1-\mathrm{r}}}\right) d r \\
& -\int_{0}^{1} \Phi^{-1}(r)\left(\frac{1}{3}\right)\left(-\frac{\mathrm{q} \ln (\mathrm{r})}{2 \sqrt{\mathrm{r}}}-\frac{\mathrm{q}}{\sqrt{\mathrm{r}}}\right. \\
& \left.+\frac{\ln (1-\mathrm{r})}{2 \mathrm{q} \sqrt{1-\mathrm{r}}}+\frac{1}{\mathrm{q} \sqrt{1-\mathrm{r}}}\right) \mathrm{dr} \\
& -\int_{0}^{1} \Psi^{-1}(r)\left(\frac{1}{3}\right)\left(-\frac{\mathrm{q} \ln (\mathrm{r})}{2 \sqrt{\mathrm{r}}}-\frac{\mathrm{q}}{\sqrt{\mathrm{r}}}\right. \\
& \left.+\frac{\ln (1-\mathrm{r})}{2 \mathrm{q} \sqrt{1-\mathrm{r}}}+\frac{1}{\mathrm{q} \sqrt{1-\mathrm{r}}}\right) \mathrm{dr} \\
= & H[\xi]+H[\eta] .
\end{aligned}
$$

Step 3. For any numbers $a \geq 0, b \geq 0$ and $q>0$ we have:

$$
H[a \xi+b \eta]=a H[\xi]+b H[\eta] .
$$

And for any real numbers $a<0, b<0$ and $q=1$, we have:

$$
H[a \xi+b \eta]=|a| H[\xi]+|b| H[\eta] .
$$

The theorem is proved.

\section{Mean-CVaR-Entropy Model for Portfolio Se- lection Problem}

Let $\boldsymbol{x}=\left(x_{1}, x_{2}, \ldots, x_{n}\right)^{T}$ be a vector of securities weight in which $x_{i}$ represent the proportion of allocation in security $i$ 
where $x_{i} \geq 0$ for $i=1,2, \ldots, n$ and $\sum_{i=1}^{n} x_{i}=1$. Let's denote the uncertain return on security $i$ by $\xi_{i}$, and the uncertain vector $\xi$ by $\boldsymbol{\xi}=\left(\xi_{1}, \xi_{2}, \ldots, \xi_{n}\right)^{T}$.

The total return for a portfolio is denoted by $\boldsymbol{x}^{T} \boldsymbol{\xi}=\sum_{i=1}^{n} x_{i} \xi_{i}$. The loss is the negative of $\boldsymbol{x}^{T} \boldsymbol{\xi}$ denoting by $f(\boldsymbol{x}, \boldsymbol{\xi})$

$$
\begin{aligned}
f(\boldsymbol{x}, \boldsymbol{\xi}) & =-\boldsymbol{x}^{T} \boldsymbol{\xi} \\
& =-\sum_{i=1}^{n} x_{i} \xi_{i}=-\left(x_{1} \xi_{1}+x_{2} \xi_{2}+\cdots+x_{n} \xi_{n}\right),
\end{aligned}
$$

according to Definition $4, V a R$ of the loss function $f(\boldsymbol{x}, \boldsymbol{\xi})$ at the confidence level $\alpha$ can be shown as

$$
\operatorname{VaR}_{f(\boldsymbol{x}, \boldsymbol{\xi})}(\alpha)=\inf \{y \mid \mathcal{M}\{f(\boldsymbol{x}, \boldsymbol{\xi}) \leq y\} \geq \alpha\},
$$

and it can be rewritten as follows:

$$
\begin{aligned}
\operatorname{VaR}_{f(\boldsymbol{x}, \boldsymbol{\xi})}(\alpha) & =\inf \{y \mid \mathcal{M}\{f(\boldsymbol{x}, \boldsymbol{\xi}) \leq y\} \geq \alpha\} \\
& =\inf \left\{y \mid \mathcal{M}\left\{-\sum_{i=1}^{n} x_{i} \xi_{i} \leq y\right\} \geq \alpha\right\} \\
& =\inf \left\{y \mid \mathcal{M}\left\{\sum_{i=1}^{n} x_{i} \xi_{i} \geq-y\right\} \geq \alpha\right\} \\
& =\inf \left\{y \mid 1-\mathcal{M}\left\{\sum_{i=1}^{n} x_{i} \xi_{i} \leq-y\right\} \geq \alpha\right\} \\
& =\inf \left\{y \mid \mathcal{M}\left\{\sum_{i=1}^{n} x_{i} \xi_{i} \leq-y\right\} \leq 1-\alpha\right\}
\end{aligned}
$$

denoting the distribution function of the total returns $\sum_{i=1}^{n} x_{i} \xi_{i}$ by $\Phi(y)=\mathcal{M}\left\{\sum_{i=1}^{n} x_{i} \xi_{i} \leq y\right\}$, we have

$$
\begin{aligned}
\operatorname{VaR}_{f(\boldsymbol{x}, \boldsymbol{\xi})}(\alpha) & =\inf \{y \mid \Phi(-y) \leq 1-\alpha\} \\
& =-\Phi^{-1}(1-\alpha) .
\end{aligned}
$$

According to Definition 5, CVaR of the loss function $f(\boldsymbol{x}, \boldsymbol{\xi})$ at the confidence level $\alpha$ can be represented as

$$
\operatorname{CVaR}_{f(\boldsymbol{x}, \boldsymbol{\xi})}(\alpha)=\frac{1}{1-\alpha} \int_{\alpha}^{1} \operatorname{VaR}_{f(\boldsymbol{x}, \boldsymbol{\xi})}(\beta) d \beta,
$$

and it can be rewritten as follows:

$$
\operatorname{CVaR}_{f(\boldsymbol{x}, \boldsymbol{\xi})}(\alpha)=\frac{1}{1-\alpha} \int_{\alpha}^{1}-\Phi^{-1}(1-\beta) d \beta .
$$

Now according to Theorem 5 we can obtain uncertainty distri- bution of portfolio returns.

Suppose that $\Phi_{1}, \Phi_{2}, \ldots, \Phi_{n}$ are continuous uncertainty distributions of uncertain security returns. Let be the uncertainty distribution of the uncertain portfolio returns $\boldsymbol{x}^{T} \boldsymbol{\xi}=\sum_{i=1}^{n} x_{i} \xi_{i}$ Since $x_{1}, x_{2}, \ldots, x_{n}$ are non-negative then the uncertainty distribution of the uncertain portfolio return can be derived as follows:

$$
\Psi^{-1}(r)=\sum_{i=1}^{n} x_{i} \Phi^{-1}(r)
$$

To maximize the investment return and to minimize the investment risk, an investor should select a portfolio which provides an optimal trade-off between expected returns and risk. If an investor is to maximize the expected return of portfolio and to minimize the investment risk simultaneously, given that entropy is greater than some preset value $c$, then the meanCVaR-entropy multi-objective portfolio selection model can be represented as follows:

$$
\left\{\begin{array}{l}
\operatorname{Max} \mathrm{E}\left[\boldsymbol{x}^{T} \boldsymbol{\xi}\right], \\
\operatorname{Min} \operatorname{CVaR}\left[\boldsymbol{x}^{T} \boldsymbol{\xi}\right] \\
\quad \text { s.t. } \\
\mathrm{H}\left[\boldsymbol{x}^{T} \boldsymbol{\xi}\right] \geq \mathrm{C}, \\
x_{1}+x_{2}+\cdots+x_{n}=1, \\
\varepsilon_{i} b_{i} \leq x_{i} \leq 1 ; \forall i=1,2, \ldots, n, \\
b_{i} \in\{0,1\} ; \forall i=1,2, \ldots, n, \\
b_{1}+b_{2}+\cdots+b_{n} \geq K .
\end{array}\right.
$$

Remark 3. $\varepsilon_{i}$ is a positive number close to zero and $K$ is the number of securities in the portfolio that the lower bound for $x_{i}$ is greater than zero.

Theorem 11. Suppose that $\xi_{1}, \xi_{2}, \ldots, \xi_{n}$ are independent skew-normal uncertain variables, denoted by $\xi_{i} \sim S N\left(e_{i}, p_{i}\right.$, $\left.\sigma_{i}\right) ; \sigma_{i}>0, p_{i}>0, i=1,2, \ldots, n$, then the equivalent model is as follows:

$$
\left\{\begin{array}{l}
\operatorname{Max} \sum_{i=1}^{n} x_{i}\left(e_{i}-\frac{\sqrt{3} \sigma_{i}}{\pi p_{i}}-\frac{\sqrt{3} \sigma_{i}}{\pi} \int_{0}^{1} \ln \left(1-r^{\frac{1}{p_{i}}}\right) d r\right), \\
\operatorname{Min}-\sum_{i=1}^{n} x_{i}\left(e_{i}+\frac{\sqrt{3} \sigma_{i}}{\pi(1-\alpha)}\left(\frac{1}{p_{i}}[(1-\alpha) \ln (1-\alpha)\right.\right. \\
\left.\left.\quad-(1-\alpha)]-\int_{0}^{1-\alpha} \ln \left(1-u^{\frac{1}{p_{i}}}\right) d u\right)\right) \\
\text { s.t. }
\end{array}\right.
$$




$$
\left\{\begin{array}{l}
-\sum_{i=1}^{n} x_{i}\left(\int_{0}^{1}\left(e_{i}+\frac{\sqrt{3} \sigma_{i}}{\pi} \ln \left(\frac{r^{\frac{1}{p_{i}}}}{1-r^{\frac{1}{p_{i}}}}\right)\right)\right. \\
\left.\quad \times\left(\frac{1}{3}\right)\left(-\frac{\mathrm{q}_{\mathrm{i}} \ln (\mathrm{r})}{2 \sqrt{\mathrm{r}}}-\frac{\mathrm{q}_{\mathrm{i}}}{\sqrt{\mathrm{r}}}+\frac{\ln (1-\mathrm{r})}{2 \mathrm{q}_{\mathrm{i}} \sqrt{1-\mathrm{r}}}+\frac{1}{\mathrm{q}_{\mathrm{i}} \sqrt{1-\mathrm{r}}}\right) \mathrm{dr}\right) \\
\geq C \\
x_{1}+x_{2}+\cdots+x_{n}=1, \\
\varepsilon_{i} b_{i} \leq x_{i} \leq 1 ; \forall i=1,2, \ldots, n, \\
b_{i} \in\{0,1\} ; \forall i=1,2, \ldots, n, \\
b_{1}+b_{2}+\cdots+b_{n} \geq K .
\end{array}\right.
$$

Proof. By applying (1) and (5) the inverse uncertainty distribution $\Phi^{-1}(r)$ of the uncertain portfolio return $\sum_{i=1}^{n} x_{i} \xi_{i}$ is derived as follows:

$$
\begin{aligned}
\Phi^{-1}(r) & =\sum_{i=1}^{n} x_{i} \Phi^{-1}(r) \\
& =\sum_{i=1}^{n} x_{i}\left(e_{i}+\frac{\sqrt{3} \sigma_{i}}{\pi} \ln \left(\frac{r^{\frac{1}{p_{i}}}}{1-r^{\frac{1}{p_{i}}}}\right)\right),
\end{aligned}
$$

where $x_{i} \geq 0, i=1,2, \ldots, n$ and $\alpha \in(0,1)$.

By using Eq. (1), Eq. (2) and Eq. (5) the expected value of the uncertain portfolio return $\boldsymbol{x}^{T} \boldsymbol{\xi}=\int_{i=1}^{n} x_{i} \xi_{i}$ is derived as follows:

$$
\begin{aligned}
& E\left[\sum_{i=1}^{n} x_{i} \xi_{i}\right] \\
& =\int_{0}^{1}\left(\sum_{i=1}^{n} x_{i}\left(e_{i}+\frac{\sqrt{3} \sigma_{i}}{\pi} \ln \left(\frac{r^{\frac{1}{p_{i}}}}{1-r^{\frac{1}{p_{i}}}}\right)\right) d r\right) \\
& =\sum_{i=1}^{n} x_{i}\left(e_{i}-\frac{\sqrt{3} \sigma_{i}}{\pi p_{i}}-\frac{\sqrt{3} \sigma_{i}}{\pi} \int_{0}^{1} \ln \left(1-r^{\frac{1}{p_{i}}}\right) d r\right) .
\end{aligned}
$$

According to Eq. (1), Eq. (3) and Eq. (5) VaR of the loss function $-\sum_{i=1}^{n} x_{i} \xi_{i}$ is

$$
\operatorname{VaR}(\alpha)=-\sum_{i=1}^{n} x_{i}\left(e_{i}+\frac{\sqrt{3} \sigma_{i}}{\pi} \ln \left(\frac{(1-\alpha)^{\frac{1}{p_{i}}}}{1-(1-\alpha)^{\frac{1}{p_{i}}}}\right)\right) .
$$

Now by using Eq. (1), Eq. (4) and Eq. (5) CVaR of the loss function $-\int_{i=1}^{n} x_{i} \xi_{i}$ is derived as follows:

\section{$\operatorname{CVaR}(\alpha)$}

$$
=\frac{1}{1-\alpha} \int_{\alpha}^{1}-\Phi^{-1}(1-\beta) d B
$$

Table 1. Uncertain return of securities

\begin{tabular}{cr}
\hline Security $\boldsymbol{i}$ & Uncertain return \\
\hline 1 & $\xi_{i} \sim S N(1.5,1,1)$ \\
2 & $\xi_{i} \sim S N(0.2,3,0.7)$ \\
$\xi_{i} \sim S N(0.6,2,1)$ \\
$\xi_{i} \sim S N(2,0.5,0.7)$ \\
$\xi_{i} \sim S N(2,0.4,0.5)$ \\
\hline 5 & $\frac{1}{1-\alpha} \int_{\alpha}^{1}-\sum_{i=1}^{n} x_{i}\left(e_{i}+\frac{\sqrt{3} \sigma_{i}}{\pi} \ln \left(\frac{(1-B)^{\frac{1}{p_{i}}}}{1-(1-B)^{\frac{1}{p_{i}}}}\right)\right) d B$ \\
$-\int_{i=1}^{n} x_{i}\left(e_{i}+\frac{\sqrt{3} \sigma_{i}}{\pi(1-\alpha)}\left(\frac{1}{p_{i}}[(1-\alpha) \ln (1-\alpha)\right.\right.$ \\
$\left.\left.-(1-\alpha)]-\int_{0}^{1-\alpha} \ln \left(1-u^{\frac{1}{p_{i}}}\right) d u\right)\right)$.
\end{tabular}

\section{Examples}

Suppose an investor is to construct portfolio containing five securities. Expert's evaluation of security returns is depicted in Table 1. The parameters $\alpha, C, K$ and lower bound vector $\varepsilon_{i}$ are designated to $0.95,1.64,3$ and $(0.02,0.02,0.02,0,0)$ by investors, respectively.

We solve the following mean-CVaR-entropy multi-objective portfolio selection problem for asymmetry entropy with different values of parameter $q$ and logarithm entropy.

$$
\left\{\begin{array}{l}
\operatorname{Max} \mathrm{E}\left[\boldsymbol{x}^{T} \boldsymbol{\xi}\right], \\
\operatorname{Min} \operatorname{CVaR}\left[\boldsymbol{x}^{T} \boldsymbol{\xi}\right] \\
\quad \text { s.t. } \\
\mathrm{H}\left[\boldsymbol{x}^{T} \boldsymbol{\xi}\right] \geq C, \\
x_{1}+x_{2}+\cdots+x_{n}=1, \\
\varepsilon_{i} b_{i} \leq x_{i} \leq 1 ; \forall i=1,2, \ldots, n, \\
b_{i} \in\{0,1\} ; \forall i=1,2, \ldots, n, \\
b_{1}+b_{2}+\cdots+b_{n} \geq K .
\end{array}\right.
$$

Table 2 is derived by solving Model (6) implementing NSGAII in MATLAB (2015b) with setting the parameter $q$ level at $0.5,1$ and 2 and logarithm entropy. As previously mentioned, portfolio diversification reduces risk by allocating investments across various securities. Furthermore, portfolio diversification cannot improve the overall expected investment return, but it can improve chances on gaining total portfolio returns closer to the expected level. Since, the optimization problem has two objective functions, a set of optimal solutions are available 
Table 2. Investment allocation of securities

\begin{tabular}{lc}
\hline \multicolumn{1}{c}{ Entropy } & Allocation \\
\hline Logarithm & $(0.5827,0.0852,0.3321,0,0)$ \\
Asymmetric $(\mathrm{q}=0.5)$ & $(0.0414,0.5535,0.4051,0,0)$ \\
Asymmetric $(\mathrm{q}=1)$ & $(0.5425,0.0213,0.4362,0,0)$ \\
Asymmetric $(\mathrm{q}=2)$ & $(0.2534,0.2737,0.4481,0.0002,0.0247)$ \\
\hline
\end{tabular}

for investors. Allocated weights can be determined based on investors' risk appetite. In this problem, in order to investigate the impact of diversification on portfolio, the optimal solution with the least $\operatorname{CVaR}\left[\boldsymbol{x}^{T} \boldsymbol{\xi}\right]$ is selected. As depicted in Table 2, a portfolio based on asymmetric entropy with $q=2$ is more diversified in comparison to asymmetric entropy with $q=0.5$ and $q=1$, and logarithm entropy.

\section{Conclusion}

In this paper, portfolio selection problem was improved via introducing skew-normal uncertainty distribution and asymmetric entropy. To show the performance of asymmetric entropy in portfolio diversification, a numerical example was presented. The example illustrated that portfolio constructed based on asymmetric entropy with $q=2$ is more diversified in comparison to asymmetric entropy with $q=0.5$ and $q=1$ and logarithm entropy. Furthermore, allocated money to securities with negative skew-normal uncertainty distributions is less than other securities.

Meanwhile, some major issues remain to be discussed. The outperformance of asymmetric entropy with $q=2$ in comparison to asymmetric entropy with $q=0.5$ and $q=1$ was investigated, which further researches are required on possible outperformance of asymmetric entropy over other forms of entropy. Moreover, applications of asymmetric entropy require to be more explored in other fields.

\section{Conflict of Interest}

No potential conflict of interest relevant to this article was reported.

\section{References}

[1] H. Markowitz, "Portfolio selection," The Journal of Finance, vol. 7, no. 1, pp. 77-91, 1952. https://doi.org/10. $2307 / 2975974$
[2] A. Sheik and H. Qiao, "Non-Normality of market returns: a framework for asset allocation decision making," The Journal of Alternative Investments, vol. 12, no. 3, pp. 8-35, 2009. https://doi.org/10.3905/JAI.2010.12.3.008

[3] F. D. Arditti, "Risk and the required return on equity," The Journal of Finance, vol. 22, no. 1, pp. 19-36, 1967. https://doi.org/10.2307/2977297

[4] F. D. Arditti and H. Levy, "Portfolio efficiency analysis in three moments: the multiperiod case," The Journal of Finance, vol. 30, no. 3, pp. 797-809, 1975. https://doi.org/ 10.1111/j.1540-6261.1975.tb01851.x

[5] P. Chunhachinda, K. Dandapani, S. Hamid, and A. J. Prakash, "Portfolio selection and skewness: Evidence from international stock markets," Journal of Banking and Finance, vol. 21, no. 2, pp. 143-167, 1997. https: //doi.org/10.1016/S0378-4266(96)00032-5

[6] H. Konno, H. Shirakawa, and H. Yamazaki, "A mean-absolute deviation-skewness portfolio optimization model," Annals of Operations Research, vol. 45, no. 1, pp. 205-220, 1993. https://doi.org/10.1007/BF02282050

[7] S. Keel, F. Herzog, H. P. Geering, and M. Mirjolet, "Optimal portfolios with skewed and heavy-tailed distributions," in Proceedings of the 3rd IASTED International Conference on Financial Engineering and Applications, Cambridge, MA, 2006, pp. 42-48.

[8] W. Hu and A. N. Kercheval, "Portfolio optimization for student $\mathrm{t}$ and skewed t returns," Quantitative Finance, vol. 10, no. 1, pp. 91-105, 2010. https://doi.org/10.1080/ 14697680902814225

[9] P. Artzner, F. Delbaen, J. M. Eber, and D. Heath, "Coherent measures of risk," Mathematical Finance, vol. 9, no. 3, pp. 203-228, 1999. https://doi.org/10.1111/14679965.00068

[10] J. Peng, "Value at risk and tail value at risk in uncertain environment," in Proceedings of the 8th International Conference on Information and Management Sciences, Kunming, China, 2009, pp. 787-793.

[11] R. T. Rockafellar and S. Uryasev, "Conditional value-atrisk for general loss distributions," Journal of Banking \& Finance, vol. 26, no. 7, pp. 1443-1471, 2002. https: //doi.org/10.1016/S0378-4266(02)00271-6 
[12] P. Jorion, "Risk ${ }^{2}$ : Measuring the risk in value at risk," $\mathrm{Fi}$ nancial Analysts Journal, vol. 52, no. 6, pp. 47-56, 1996. https://doi.org/10.2469/faj.v52.n6.2039

[13] N. Krejic, M. Kumaresan, and A. Roznjik, "VaR optimal portfolio with transaction costs," Applied Mathematics and Computation, vol. 218, no. 8, pp. 4626-4637, 2011. https://doi.org/10.1016/j.amc.2011.10.047

[14] R. Mansini, W. Ogryczak, and M. G. Speranza, "Conditional value at risk and related linear programming models for portfolio optimization," Annals of Operations Research, vol. 152, no. 1, pp. 227-256, 2007. https: //doi.org/10.1007/s10479-006-0142-4

[15] X. Tong, L. Qi, F. Wu, and H. Zhou, "A smoothing method for solving portfolio optimization with CVaR and applications in allocation of generation asset," Applied Mathematics and Computation, vol. 216, no. 6, pp. 1723-1740, 2010. https://doi.org/10.1016/j.amc.2009.12.031

[16] A. A. Najafi and S. Mushakhian, "Multi-stage stochastic mean-semivariance-CVaR portfolio optimization under transaction costs," Applied Mathematics and Computation, vol. 256, pp. 445-458, 2015. https://doi.org/10.1016/ j.amc.2015.01.050

[17] C. E. Shannon, "A mathematical theory of communication," The Bell System Technical Journal, vol. 27, no. 3, pp. 379-423, 1948. https://doi.org/10.1002/j.1538-7305. 1948.tb01338.x

[18] G. C. Philippatos and C. J. Wilson, "Entropy, market risk, and the selection of efficient portfolios," Applied Economics, vol. 4, no. 3, pp. 209-220, 1972. https://doi. org/10.1080/00036847200000017

[19] M. R. Simonelli, "Indeterminacy in portfolio selection," European Journal of Operational Research, vol. 163, no. 1, pp. 170-176, 2005. https://doi.org/10.1016/j. ejor.2004.01.006

[20] A. P. Jacquemin and C. H. Berry, "Entropy measure of diversification and corporate growth," The Journal of Industrial Economics, vol. 27, no. 4, pp. 359-369, 1979. https://doi.org/10.2307/2097958

[21] R. E. Hoskisson, M. A. Hitt, R. A. Johnson, and D. D. Moesel, "Construct validity of an objective (entropy) categorical measure of diversification strategy," Strategic
Management Journal, vol. 14, no. 3, pp. 215-235, 1993. https://doi.org/10.1002/smj.4250140305

[22] A. K. Bera and S. Y. Park, "Optimal portfolio diversification using the maximum entropy principle," Econometric Reviews, vol. 27, no. 4-6, pp. 484-512, 2008. https://doi.org/10.1080/07474930801960394

[23] B. Liu, "Why is there a need for uncertainty theory?," Journal of Uncertain Systems, vol. 6, no. 1, pp. 3-10, 2012.

[24] B. Liu, Uncertainty Theory, 5th ed. Heidelberg, Germany: Springer, 2018.

[25] B. Liu, Uncertainty Theory, 2nd ed. Heidelberg, Germany: Springer, 2007.

[26] J. Zhai, M. Bai, and H. Wu, "Mean-risk-skewness models for portfolio optimization based on uncertain measure," Optimization, vol. 67, no. 5, pp. 701-714, 2018. https: //doi.org/10.1080/02331934.2018.1426577

[27] R. Bhattacharyya, A. Chatterjee, and S. Kar, "Meanvariance-skewness portfolio selection model in general uncertain environment," Indian Journal of Industrial and Applied Mathematics, vol. 3, no. 1, pp. 45-61, 2012.

[28] L. Yan, "Mean-VaR model for uncertain portfolio selection," Journal of Information \&Computational Science, vol. 8, no. 15, pp. 3523-3530, 2011.

[29] Y. Ning, L. Yan, and Y. Xie, "Mean-TVaR model for portfolio selection with uncertain returns," Information (Tokyo), vol. 16, no. 2, pp. 977-986, 2013.

[30] B. Liu, "Some research problems in uncertainty theory," Journal of Uncertain Systems, vol. 3, no. 1, pp. 3-10, 2009.

[31] X. Chen and W. Dai, "Maximum entropy principle for uncertain variables," International Journal of Fuzzy Systems, vol. 13, no. 3, pp. 232-236, 2011.

[32] W. Dai and X. Chen, "Entropy of function of uncertain variables," Mathematical and Computer Modelling, vol. 55, no. 3-4, pp. 754-760, 2012. https://doi.org/10.1016/j. mcm.2011.08.052

[33] A. Sajedi and G. Yari, "Order $\nu$ entropy and cross entropy of uncertain variables for portfolio selection," International Journal of Fuzzy Logic and Intelligent Systems, vol. 
20, no.1, pp. 35-42, 2020. http://doi.org/10.5391/IJFIS. 2020.20.1.35

[34] K. Deb, S. Agrawal, A. Pratap, and T. Meyarivan, "A fast elitist non-dominated sorting genetic algorithm for multiobjective optimization: NSGA-II," in Parallel Problem Solving from Nature - PPSN VI. Heidelberg, Germany: Springer, 2020, pp. 849-858. https://doi.org/10.1007/3540-45356-3_83

[35] J. Kennedy and R. Eberhart, "Particle swarm optimization," in Proceedings of the International Conference on Neural Networks (ICNN), Perth, Australia, 1995, pp. 1942-1948. https://doi.org/10.1109/ICNN.1995.488968

[36] C. M. Fonseca and P. J. Fleming, "Genetic Algorithms for multiobjective optimization: formulationdiscussion and generalization," in Proceedings of the 5th International Conference on Genetic Algorithms, Urbana-Champaign, IL, 1993, pp. 416-423.

[37] E. Zitzler, K. Deb, and L. Thiele, "Comparison of multiobjective evolutionary algorithms: empirical results," Evolutionary Computation, vol. 8, no. 2, pp. 173-195, 2000. https://doi.org/10.1162/106365600568202

[38] Z. Sherinov and A. Unveren, "Multi-objective imperialistic competitive algorithm with multiple non-dominated sets for the solution of global optimization problems," Soft Computing, vol. 22, no. 24, pp. 8273-8288, 2018. https: //doi.org/10.1007/s00500-017-2773-6

[39] E. Atashpaz-Gargari and C. Lucas, "Imperialist competitive algorithm: an algorithm for optimization inspired by imperialistic competition," in Proceedings of 2007 IEEE Congress on Evolutionary Computation, Singapore, 2007, pp. 4661-4667. https://doi.org/10.1109/CEC.2007. 4425083

[40] Y. Liu and W. Lio, "A revision of sufficient and necessary condition of uncertainty distribution," Journal of Intelligent \& Fuzzy Systems, vol. 38, no. 4, pp. 4845-4854, 2020. https://doi.org/10.3233/JIFS-191535

[41] B. Liu, "Uncertainty theory," in Uncertainty Theory. Heidelberg, Germany: Springer, 2010, pp. 1-79. https: //doi.org/10.1007/978-3-642-13959-8_1

[42] B. Liu, "Toward uncertain finance theory," Journal of Uncertainty Analysis and Applications, vol. 1, article no. 1, 2013. https://doi.org/10.1186/2195-5468-1-1

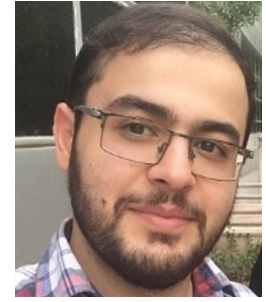

Seyyed Hamed Abtahi is a PhD candidate in Statistics in the Department of Statistics at Science and Research Branch, Islamic Azad University (SRBIAU). He received his M.Sc. in Mathematical Statistics from Allameh Tabataba'i University in 2014. His research interests include uncertainty theory, portfolio selection and entropy.

E-mail:

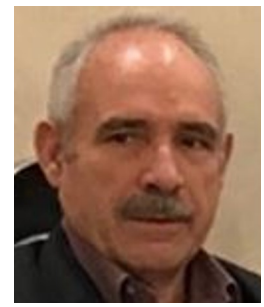

Gholamhossein Yari is an associate professor in the department of Mathematics at Iran University of Science \& Technology. Dr. Yari received his Ph.D. in Applied Mathematics and Statistics in 2004 from Iran University of Science \& Technology. His major researches include information theory and stochastic processes.

E-mail:

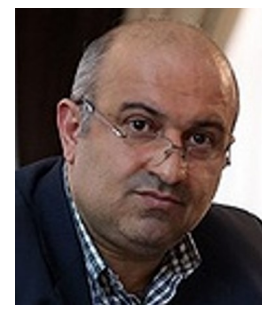

Farhad Hosseinzadeh Lotfi is a professor of Mathematics in the Department of Mathematics at Islamic Azad University, Science and Research Branch (SRBIAU). He received his Ph.D. in Applied Mathematics from Science and Research Branch, Islamic Azad University (SRBIAU) in 2000. His research focuses on Operation Research and DEA.

E-mail:

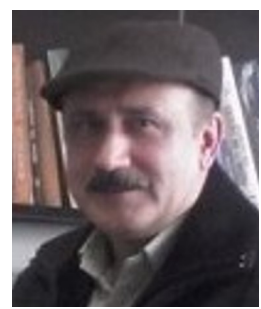

Rahman Farnoosh is a Professor of Applied Statistics in the Department of Mathematics at Iran University of Science \& Technology. He received his Ph.D. in Statistics from Leeds University in 2000. His research interests are financial mathematics, image analysis and stochastic differential equation. E-mail: 